\title{
Análisis arqueométrico de pigmentos del sitio arqueológico Tambo Colorado por difracción de rayos $X$ sincrotrón
}

\author{
Archaeometrical analysis of pigments from \\ Tambo Colorado archaeological site by \\ synchrotron X-ray diffraction
}

Elvira Zeballos-Velásquez ${ }^{1}$, Véronique Wright ${ }^{2}$, Leopoldo Suescun ${ }^{3}$, Esteban Asto ${ }^{1}$

\footnotetext{
${ }^{1}$ Laboratorio de Cristalografía. Facultad de Ciencias Físicas, Universidad Nacional Mayor de San Marcos, Lima, Lima, Perú.

${ }^{2}$ Ministère des Affaires Étrangères et du Développement International (MAEDI), Institut Français d'Études Andines (IFEA), UMIFRE 17 - CNRS MAE USR3337, Projet de Recherche Tambo Colorado, Lima, Lima, Peru

${ }^{3}$ Cryssmat-Lab / Cátedra de Física / DETEMA, Facultad de Química, Universidad de la República, Montevideo, Montevideo, Uruguay.

e-mail: ezeballosv@unmsm.edu.pe,vero_wright@ @otmail.com, leopoldosuescun@gmail.com, elvis27anis@gmail.com
}

\section{RESUMEN}

A pesar de la excepcional arquitectura y policromía del sitio arqueológico de Tambo Colorado, no ha habido trabajos de conservación ni en las estructuras arquitectónicas de tierra cruda, ni en las superficies. Estos trabajos requieren una comprensión adecuada del estado de conservación de la arquitectura del sitio y el análisis de los materiales utilizados. Con este propósito, los resultados del presente estudio pretenden explicar la naturaleza de los materiales (pigmentos) utilizados en las pinturas murales, así como determinar las fuentes de estos materiales (canteras). Para ello, pigmentos de cantera fueron investigados por la técnica de difracción de rayos X, usando radiación sincrotrón, y refinamiento estructural por el método de Rietveld. Se determinó la composición de los pigmentos, identificando las fases de anortita, hematita, cuarzo, clorita, halita, yeso, ortoclasa, illita, jarosita, calcita, caolinita, zinwaldita, cristobalita, anhidrita y albita. El refinamiento Rietveld confirmó la presencia de estas fases y determinó el porcentaje en peso de cada una de ellas.

Palabras-llave: Pigmento, difracción de rayos-X, método de Rietveld, radiación sincrotrón.

\begin{abstract}
Despite the exceptional architecture and polychromy of archaeological site of Tambo Colorado, there has been no conservation work or in the architectural structures of raw earth, nor on the surfaces. These jobs require a proper understanding of the state of conservation of the site architecture and the analysis of the materials used. With this purpose, the results of the present study tries to explain the nature of the materials (pigments) used in the mural paintings, as well as determine the sources of these materials (quarries). To do this, pigments of quarry were investigated by the technique of X-ray diffraction, using synchrotron radiation, and structural refinement by the Rietveld method. It was determined the composition of pigments, identifying the phases of anorthite, quartz, hematite, chlorite, halite, gypsum, orthoclase, illite, jarosite, calcite, kaolinite, zinwaldite, cristobalite, anhydrite and albite. Rietveld refinement confirmed the presence of these phases and determined the percentage by weight of each of them.
\end{abstract}

Keywords: Pigments, X-ray diffraction, Rietveld method, synchrotron radiation.

\section{INTRODUCCIÓN}

El complejo arqueológico Tambo Colorado se encuentra ubicado en la margen derecha del valle de Pisco, cerca del pueblo de Humay, a $290 \mathrm{Km}$ al sur de Lima y a $35 \mathrm{Km}$ de la ciudad de Pisco (Figura 1). También llamado Pucallacta o Pucahuasi (de puca que significa "rojo" en quechua), fue edificado en el camino lateral principal del QhapaqNan que conecta el camino de la sierra con el camino principal de la costa. 


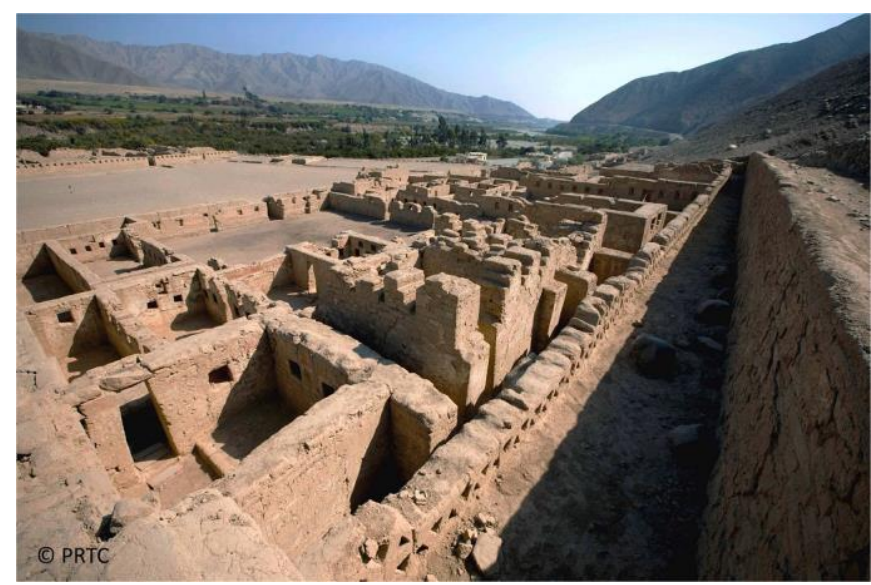

Figura 1: Tambo Colorado. Construcción de adobe con trazado típico inca.

Este complejo es uno de los sitios arqueológicos más impresionantes de la costa sur peruana, debido a su imponente arquitectura, a su extraordinaria decoración pictórica y a su estratégica ubicación en el tramo del gran Camino Inca. Todas estas características se encuentran estrechamente vinculadas con el papel que este sitio jugó dentro del proceso de la conquista Inca en la costa.

No obstante la extraordinaria arquitectura y policromía de Tambo Colorado y su evidente velocidad de degradación, no se han realizado trabajos de conservación ni en las estructuras arquitectónicas de tierra cruda, ni en las superficies (pintura mural, enlucidos), hecho contradictorio con las publicaciones que mencionan se trata de uno de los sitios incas mejor conservados del Perú.

En el marco de esta problemática, análisis estructurales de los materiales policromos fueron realizadas en el 2014, utilizando técnicas físicas [1]. Sus resultados (Tabla 1) indican una correspondencia en la composición de base de los pigmentos arqueológicos (MM) y geológicos (MC), pero con una diferencia debida a la presencia de sales (halita) en la cantera (área geológica ubicada aproximadamente a $300 \mathrm{~m}$ al norte del complejo). Estos componentes corresponderían a una contaminación exterior, lo que en el presente trabajo ha sido estudiado analizando muestras geológicas extraídas de vetas más profundas de la cantera.

Tabla 1: Porcentaje en peso de las fases en pigmentos geológicos MC y pigmentos arqueológicos MM.

\begin{tabular}{l|c|c|c|c|c|c}
\hline \multirow{2}{*}{ FASES } & \multicolumn{6}{|c}{ MUESTRAS } \\
\cline { 2 - 7 } & \multicolumn{3}{|c}{ MUESTRAS MC $^{*}$} & \multicolumn{3}{c}{ MUESTRAS MM $^{* *}$} \\
\cline { 2 - 7 } & $\begin{array}{c}\text { M-2 } \\
\text { Rojo }\end{array}$ & $\begin{array}{c}\text { M-3 } \\
\text { Amarillo }\end{array}$ & $\begin{array}{c}\text { M-4 } \\
\text { Blanco }\end{array}$ & $\begin{array}{c}\text { M-9 } \\
\text { Rojo }\end{array}$ & $\begin{array}{c}\text { M-11 } \\
\text { Amarillo }\end{array}$ & $\begin{array}{c}\text { M-12 } \\
\text { Blanco }\end{array}$ \\
\hline Cuarzo & 7.61 & 81.86 & 36.93 & 39.68 & 34.76 & 37.04 \\
\hline Anorthita & 13.28 & & 22.06 & 26.13 & 25.71 & 14.31 \\
\hline Chlorita & 0.22 & & 0.60 & 0.60 & 0.89 & 2.06 \\
\hline Halita & 60.18 & 0.81 & 26.20 & & & \\
\hline Yeso & 4.14 & 2.74 & 3.31 & 9.20 & 9.70 & 11.10 \\
\hline Illita & 2.71 & 7.63 & 6.53 & 8.18 & 5.51 & 7.20 \\
\hline Albita & & & & 13.49 & 19.80 & 21.24 \\
\hline Jarosita & 11.86 & 6.95 & 4.38 & 2.71 & 3.63 & 7.05 \\
\hline
\end{tabular}

* Pigmentos geológicos (muestras extraídas de la superfície de la cantera).

** Pigmentos arqueológicos (muestras de mural). 
Las muestras del presente trabajo fueron investigadas por Difracción de Rayos-X [2], utilizando radiación sincrotrón (DRXS) [3], para determinar su composición. Las medidas DRXS fueron analizadas por el Método de Rietveld [4-7] utilizando el programa computacional TOPAS [8,9], a fin de determinar el porcentaje en peso de cada fase. Los resultados han proporcionado información cualitativa y cuantitativa importante acerca de la composición de las muestras.

\section{MATERIALES Y MÉTODOS}

Las muestras estudiadas en el presente trabajo fueron extraídas de vetas más profundas de la cantera investigada en trabajo anterior [1] , a fin de derterminar la influencia del medio ambiente en la composición de las muestras por su contenido de halita. La composición mineralógica de las muestras, previamente pulverizadas, fue estudiada por la técnica de Difracción de rayos-X, usando radiación sincrotrón. Datos de difracción de polvo de alta resolución de radiación sincrotrón se recogieron en todas las muestras, a temperatura ambiente, usando la línea de luz 11-BM del Advanced Photon Source (APS), del Argonne National Laboratory, con una longitud de onda incidente de $0.414176 \AA$. Las muestras en polvo se depositaron en capilares de poliamida de 0,8 $\mathrm{mm}$ de diámetro, que se hizo girar a $10 \mathrm{~Hz}$ durante la colecta de datos. Los datos fueron recolectados a través de doce cristales analizadores de $\mathrm{Si}$ (111) de la línea de luz, junto a doce cintiladores Oxford-Danfysik $\mathrm{LaCl}$, que se escanearon en un rango $2 \theta$ de 0.8 a 50 grados, con paso angular de $0,001^{\circ} 2 \theta$ y 0,1 seg. por paso.

Las medidas fueron analizadas por el Método de Rietveld, a fin de determinar cuantitativamente la composición de las fases.

\section{RESULTADOS Y DISCUSIÓN}

La Figura 2 muestra los difractogramas (hasta 20:20 ${ }^{\circ}$ ) correspondientes a medidas DRXS de las muestras TC-1 (pigmento rojo), TC-2 (pigmento blanco), TC-3 (pigmento gris), TC-4 (pigmento amarillo), TC-5 (pigmento gris brillante) y TC-6 (pigmento verde).

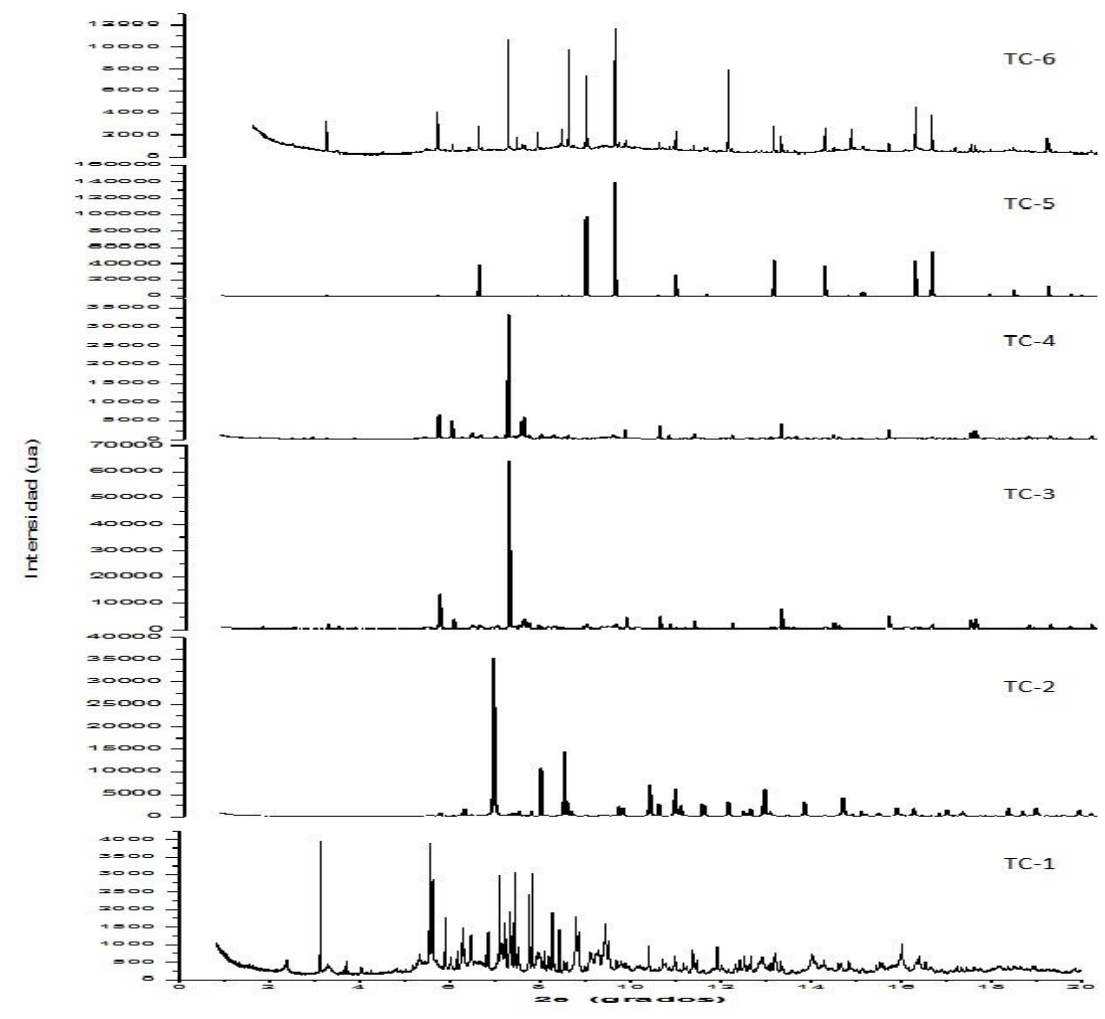

Figura 2: Difractogramas de las muestras TC-1, TC-2, TC-3, TC-4, TC-5 y TC-6. 
La identificación de fases de cada muestra, a partir del análisis de las medidas de DRXS, fue realizada con el programa EVA de Bruker-AXS. En la Figura 3 mostramos las fases identificadas para la muestra TC-1 y en la Tabla 2 presentamos las fases identificadas en todas las muestras.

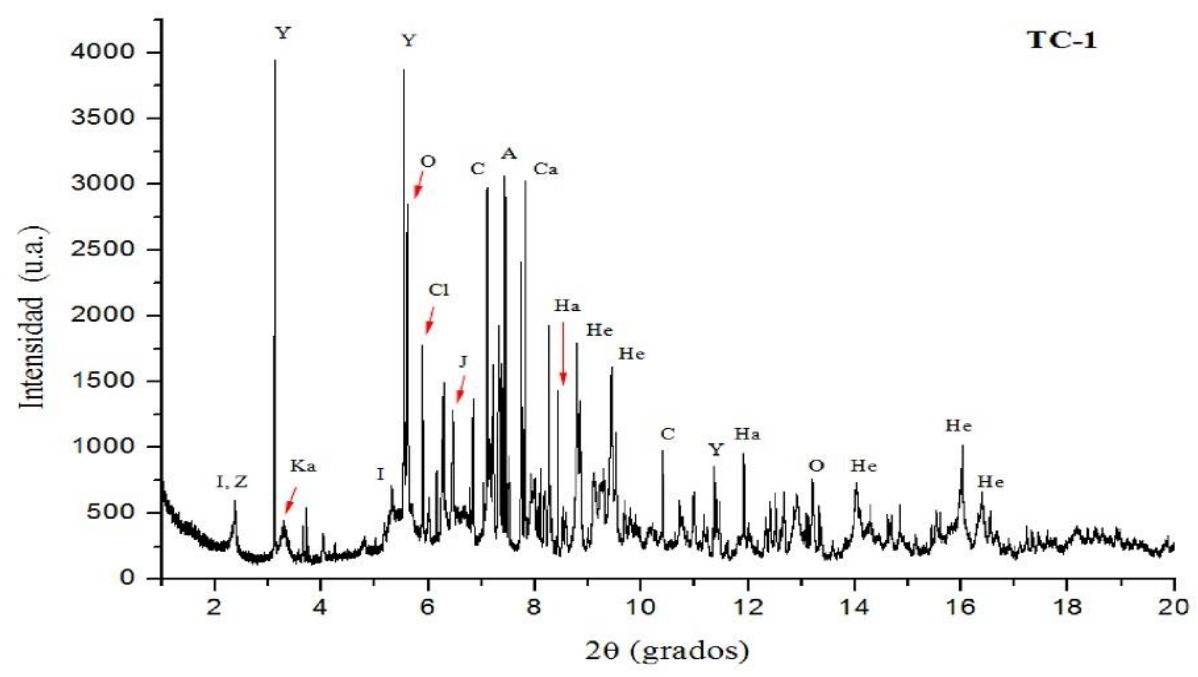

Figura 3: Fases identificadas en la muestra TC-1. (I: illita; Z: zinnwualdita; Y: yeso; Ka: caolinita; O: ortoclasa; Cl: clorita; J: jarosita; C: cuarzo; A: anortita; Ca: calcita; Ha: halita; He: hematita.

Tabla 2: Fases identificadas en cada muestra.

\begin{tabular}{|c|c|c|c|c|c|c|c|}
\hline \multirow[t]{2}{*}{ FASES } & \multicolumn{6}{|c|}{ MUESTRAS } & \multirow[t]{2}{*}{ ICSD } \\
\hline & $\begin{array}{c}\text { TC-1 } \\
\text { (Rojo) }\end{array}$ & $\begin{array}{c}\text { TC-2 } \\
\text { (Blanco) }\end{array}$ & $\begin{array}{l}\text { TC-3 } \\
\text { (Gris) }\end{array}$ & $\begin{array}{c}\text { TC-4 } \\
\text { (Amarillo) }\end{array}$ & $\begin{array}{c}\text { TC-5 } \\
\text { (Gris brillante) }\end{array}$ & $\begin{array}{c}\text { TC-6 } \\
\text { (Verde) }\end{array}$ & \\
\hline Cuarzo & $\checkmark$ & & $\checkmark$ & $\checkmark$ & $\checkmark$ & $\checkmark$ & 027826 \\
\hline Illita & $\checkmark$ & $\checkmark$ & $\checkmark$ & $\checkmark$ & & $\checkmark$ & 090144 \\
\hline Caolinita & $\checkmark$ & & & & & & 087771 \\
\hline Clorita & $\checkmark$ & & $\checkmark$ & $\checkmark$ & & $\checkmark$ & 156166 \\
\hline Anortita & $\checkmark$ & & $\checkmark$ & $\checkmark$ & & & 000654 \\
\hline Yeso & $\checkmark$ & & $\checkmark$ & $\checkmark$ & $\checkmark$ & $\checkmark$ & 002057 \\
\hline Hematita & $\checkmark$ & $\checkmark$ & $\checkmark$ & & $\checkmark$ & $\checkmark$ & 015840 \\
\hline Halita & $\checkmark$ & $\checkmark$ & & & $\checkmark$ & $\checkmark$ & 028948 \\
\hline Ortoclasa & $\checkmark$ & $\checkmark$ & $\checkmark$ & $\checkmark$ & & & 009544 \\
\hline Zinnwaldita & $\checkmark$ & & & & & & 010401 \\
\hline Calcita & $\checkmark$ & $\checkmark$ & & & & & 018164 \\
\hline Jarosita & $\checkmark$ & & & & & & 034344 \\
\hline Anhidrita & & $\checkmark$ & & & & & 040043 \\
\hline Albita & & $\checkmark$ & $\checkmark$ & $\checkmark$ & & $\checkmark$ & 087654 \\
\hline Cristobalita & & & $\checkmark$ & $\checkmark$ & & $\checkmark$ & 034927 \\
\hline
\end{tabular}


Estos resultados fueron confirmados por refinamiento estructural con el método de Rietveld. Todas las fases identificadas fueron refinadas estructuralmente para determinar su porcentaje en peso. Para el ajuste usamos la función PV-TCHZ (pseudo-Voigt Thompson-Cox-Hastings). Las Figuras 4 y 5 presentan los resultados del refinamiento estructural a partir del modelamiento de los difractogramas de todas las muestras.

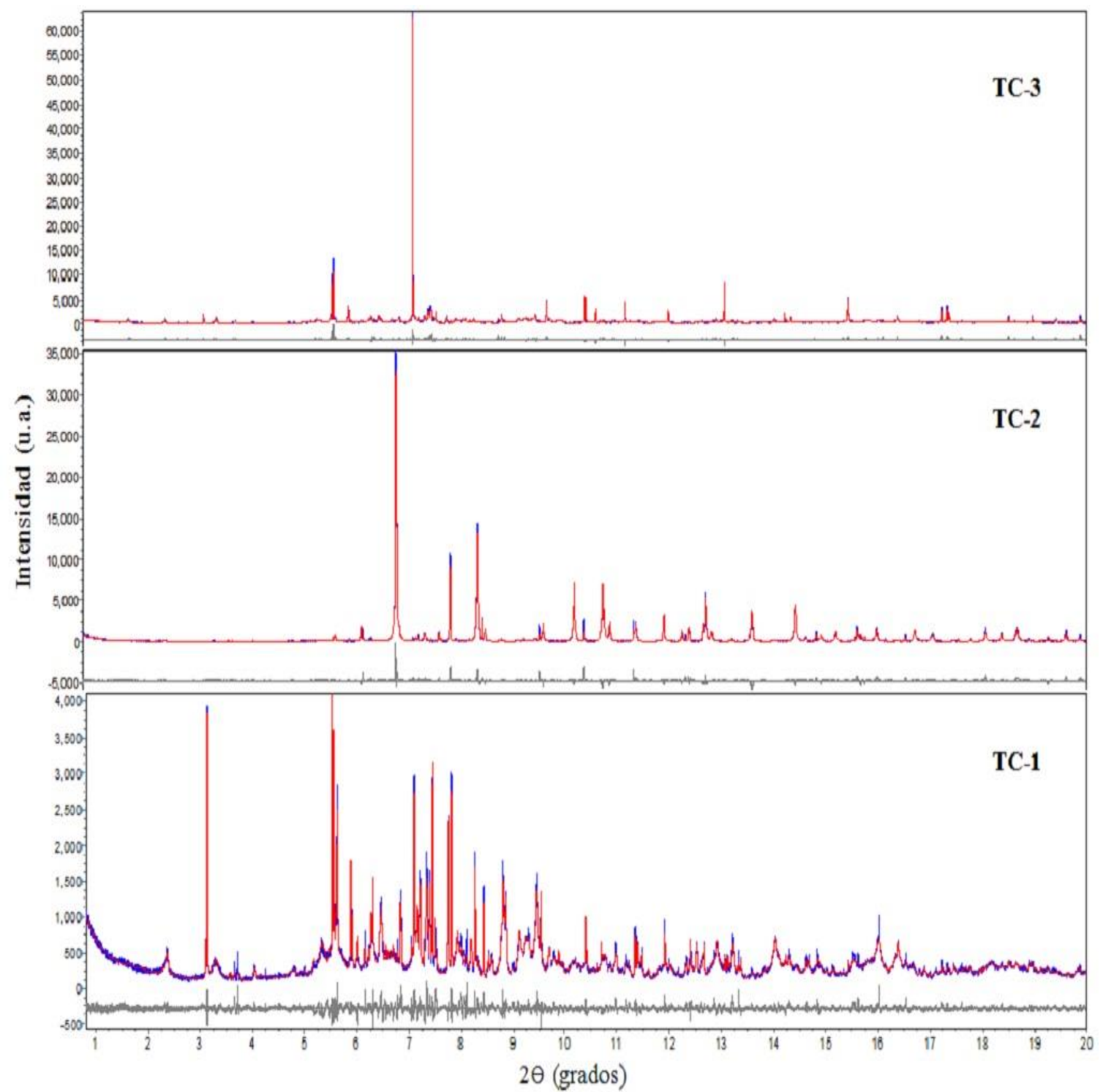

Figura 4: Refinamiento estructural correspondiente a las muestras TC-1, TC-2 y TC-3. 


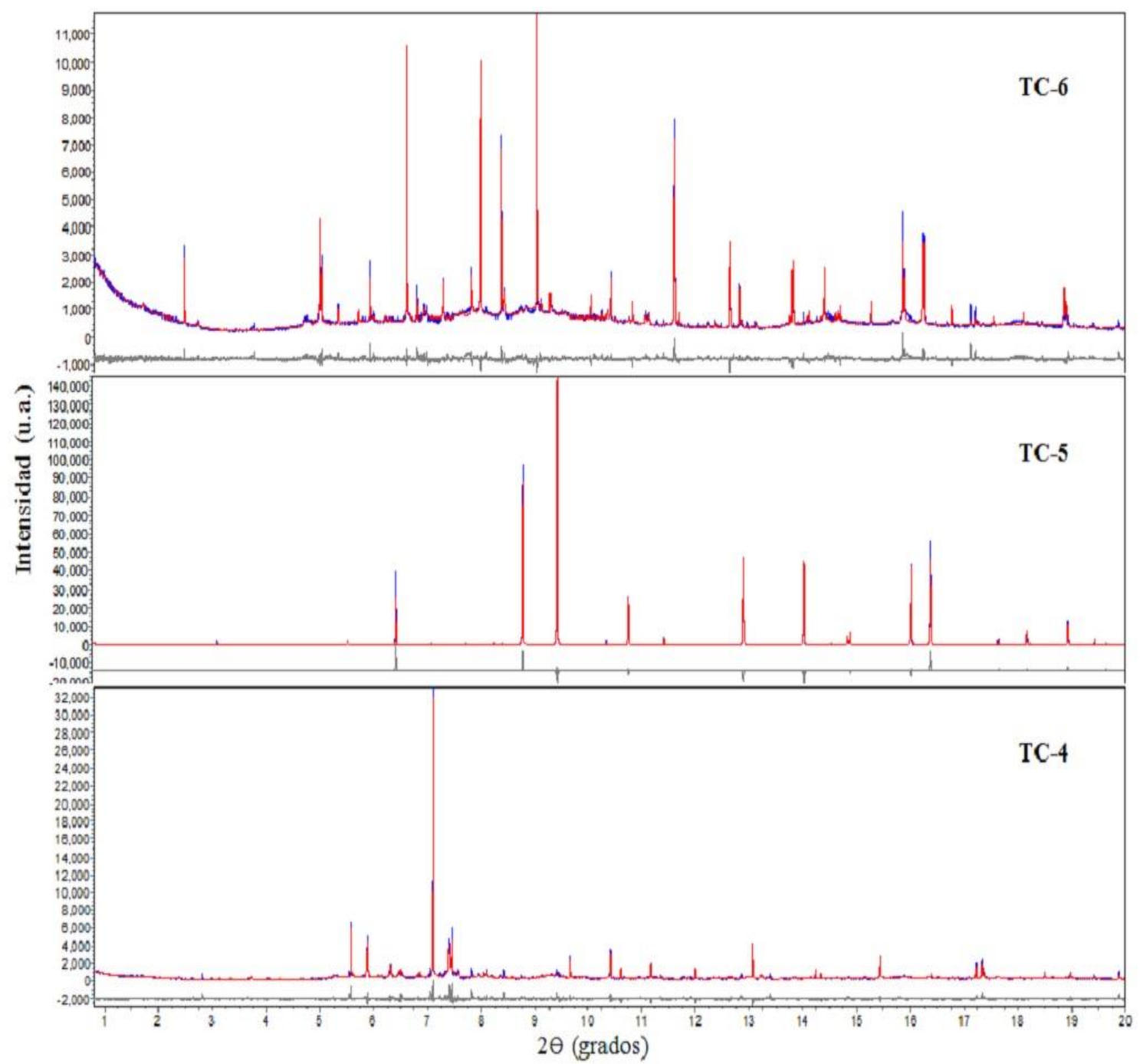

Figura 5: Refinamiento estructural correspondiente a las muestras TC-4, TC-5 y TC-6.

Los valores de los índices de ajuste $R_{\mathrm{wp}}$ y $R$ exp del refinamiento son mostrados en la Tabla 3. En la Tabla 4 mostramos el porcentaje en peso de cada fase.

Tabla 3: Índices $R$ de refinamiento.

\begin{tabular}{c|c|c}
\hline \multirow{2}{*}{ MUESTRAS } & \multicolumn{2}{|c}{ ÍNDICES DE AJUSTE (\%) } \\
\cline { 2 - 3 } & $\mathbf{R}_{\text {EXP }}$ & $\mathbf{R}_{\mathrm{WP}}$ \\
\hline TC-1 & 5.19 & 10.03 \\
\hline TC-2 & 4.63 & 15.07 \\
\hline TC-3 & 4.40 & 14.89 \\
\hline TC-4 & 4.72 & 15.83 \\
\hline TC-5 & 4.31 & 15.74 \\
\hline TC-6 & 3.83 & 7.90 \\
\hline
\end{tabular}


Tabla 4: Porcentaje en peso de las fases.

\begin{tabular}{|c|c|c|c|c|c|c|}
\hline \multirow[t]{2}{*}{ FASES } & \multicolumn{6}{|c|}{ MUESTRAS } \\
\hline & $\begin{array}{l}\text { TC-1 } \\
\text { (Rojo) }\end{array}$ & $\begin{array}{c}\text { TC-2 } \\
\text { (Blanco) }\end{array}$ & $\begin{array}{l}\text { TC-3 } \\
\text { (Gris) }\end{array}$ & $\begin{array}{c}\text { TC-4 } \\
\text { (Amarillo) }\end{array}$ & $\begin{array}{c}\text { TC-5 } \\
\text { (Gris brillante) }\end{array}$ & $\begin{array}{c}\text { TC-6 } \\
\text { (Verde) }\end{array}$ \\
\hline Cuarzo & 3.98 & & 30.26 & 24.91 & 0.82 & 29.05 \\
\hline Illita & 8.18 & 2.11 & 3.60 & 5.45 & & 9.81 \\
\hline Caolinita & 5.11 & & & & & \\
\hline Clorita & 1.14 & & 17.51 & 11.31 & & 12.60 \\
\hline Anortita & 10.55 & & 1.85 & 3.74 & & \\
\hline Yeso & 31.47 & & 1.70 & 1.43 & 12.49 & 18.43 \\
\hline Hematita & 13.75 & 0.54 & 1.09 & & 85.81 & 9.70 \\
\hline Halita & 2.85 & 1.51 & & & 0.88 & 9.96 \\
\hline Ortoclasa & 5.10 & 3.26 & 15.43 & 2.71 & & \\
\hline Zinnwaldita & 1.45 & & & & & \\
\hline Calcita & 14.74 & 3.32 & & & & \\
\hline Jarosita & 1.69 & & & & & \\
\hline Anhidrita & & 88.59 & & & & \\
\hline Albita & & 0.67 & 26.78 & 38.64 & & 9.91 \\
\hline Cristobalita & & & 1.78 & 11.81 & & 0.53 \\
\hline
\end{tabular}

Analizando estos resultados con los obtenidos en estudio anterior [1] (ver Tabla 1), se observa que los pigmentos rojo (TC-1) y amarillo (TC-4) contienen las mismas fases de los pigmentos rojo (M-9) y amarillo (M-11) utilizados en los murales, lo que induce a concluir que estos últimos procederían de la cantera investigada, confirmando resultados del estudio anterior.

Los pigmentos de cantera rojo y blanco presentan fase de halita en muy bajo porcentaje $(2.85 \%$ y $1.51 \%$, respectivamente), en relación a los porcentajes de las muestras extraídas de capas superficiales de la misma cantera $(60.18 \%$ y $26.20 \%$, respectivamente) investigadas anteriormente [1]. El pigmento amarillo no presenta fase de halita, en tanto que en pigmento amarillo proveniente de capas superficiales de la misma cantera fue determinada la presencia de halita, aunque en bajo porcentaje $(0.81 \%)$.

\section{CONCLUSIONES}

Fueron evaluadas muestras de pigmento geológico rojo, amarillo, blanco, gris, gris brillante y verde, extraídas de vetas más profundas de cantera. El análisis cualitativo reportó la presencia de cuarzo, anortita, hematita, clorita, halita, yeso, ortoclasa, illita, jarosita, calcita, caolinita, zinwaldita, cristobalita, anhidrita y albita en las muestras.

Los pigmentos geológicos rojo y amarillo contienen las fases presentes en los pigmentos rojo y amarillo arqueológicos, lo cual indicaría que estos últimos procederían de la cantera investigada. Asimismo, los pigmentos rojo y blanco contienen halita en porcentaje mucho menor que el de las muestras superficiales estudiadas anteriormente; además, el pigmento amarillo no contiene halita. Estos resultados, donde la halita está drásticamente disminuida o ausente, estarían confirmando la hipótesis formulada en el presente estudio; es decir, la presencia de halita se debería a contaminación exterior, cuya proporción es menor en capas más profundas de la cantera.

Para estudios arqueométricos por difracción de rayos X de muestras en polvo, la metodología con radiación sincrotrón resulta particularmente muy eficaz, no sólo por sus ventajas frente a la radiación convencional, sino también porque se requiere mucho menos cantidad de muestra. 


\section{AGRADECIMIENTOS}

Nuestro agradecimiento al Argonne National Laboratory por su contribución con las medidas sincrotrón, así como al Vicerrectorado de Investigación y Posgrado de la Universidad Nacional Mayor de San Marcos por el soporte financiero.

Use of the Advanced Photon Source at Argonne National Laboratory was supported by the U. S. Department of Energy, Office of Science, Office of Basic Energy Sciences, under Contract No. DE-AC02$06 \mathrm{CH} 11357$.

\section{BIBLIOGRAFÍA}

[1] WRIGHT V., PACHECO G., TORRES H., et al., "Mural Paintings in Ancient Peru: The Case of Tambo Colorado, Pisco Valley", STAR: Science and Technology of Archaeological Research, v. 1, n. 2, pp. 11-20, 2016.

[2] MOORE, M. M., REYNOLDS Jr., R. C. X-ray Diffraction and the Identification and Analysis of Clay Minerals, 2 ed., Oxford University Press, Oxford, 1997.

[3] LEE, P.L., SHU, D., RAMANATHAN, M., et al., "A twelve-analyzer detector system for high-resolution powder diffraction", J. Synchrotron Rad., v. 15, pp. 427-432, 2008.

[4] YOUNG, R.A., LARSON, A.C., PAIVA-SANTOS, C.O., User's guide to program dbws-9807a for Rietveld analysis of X-ray and neutron powder diffraction patterns with a PC and various other computers. School of Physics, Georgia Institute of Technology, Atlanta, GA, EUA., 2000.

[5] FANCIO E. Aplicação do Metodo de Rietveld para Análise Quantitativa de Fases dos polimorfos da Zircônia por Difração de Raios X, Tese de D.Sc., Instituto de Pesquisas Energéticas e Nucleares Universidade de São Paulo, 1999.

[6] RODRÍGUEZ-CARVAJAL, J. "FULLPROF: A Program for Rietveld Refinement and Pattern Matching Analysis", In: Satellite Meeting on Powder Diffraction of the XV Congress of the IUCr, Abstracts, p. 127, Toulouse, 1990.

[7] RIETVELD, H.M. “A Profile Refinement Method for Nuclear and Magnetic”, J.Appl. Cryst., v.2, pp. 6571, 1969.

[8] BRUKER AXS GMBH. TOPAS. User's Manual, 2009.

[9] DIFFRACplus. TOPAS/TOPAS R/TOPAS P. Version 3.0. Technical Reference, BRUKER AXS GmbH, Karlsruhe, West Germany, 2005.

\section{ORCID}

Elvira Zeballos-Velásquez

https://orcid.org/0000-0002-5957-1639

Véronique Wright

Leopoldo Suescun

https://orcid.org/0000-0002-0487-5483

Esteban Asto

https://orcid.org/0000-0002-7606-8074

https://orcid.org/0000-0003-4366-6091 